\title{
Gain dispersion induced subpicosecond pulse breakup in a fiber and semiconductor laser amplifier combined system
}

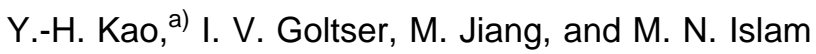 \\ The University of Michigan, Ann Arbor, Michigan 48109 \\ G. Raybon \\ Lucent Technologies, Holmdel, New Jersey 07733 \\ (Received 24 September 1996; accepted for publication 29 October 1996)

\begin{abstract}
We observe pulse breakup for 650-fs pulses propagating through $9 \mathrm{~m}$ of optical fiber followed by an InGaAsP amplifier. The pulses are broadened by a factor of two, and a second peak appears at about 2 ps after the main peak. To identify the responsible mechanisms, we solve numerically the propagation equations including nonlinear carrier dynamics and gain dispersion. We attribute the broadening to two photon absorption and the breakup to the interplay between linear gain dispersion and frequency chirp in the amplifier. These pulse distortions could impact devices involving fibers and semiconductor amplifiers for high speed $(>200 \mathrm{~Gb} / \mathrm{s})$ optical switching or transmission.

(C) 1996 American Institute of Physics. [S0003-6951(96)03853-3]
\end{abstract}

Ultrafast compact optical switching devices are the key elements in high speed communication networks. Semiconductor laser amplifiers (SLAs) have been considered as promising candidates for high speed all-optical switching devices because of their high nonlinearities (at least four orders of magnitude higher than optical fibers), which lead to compactness and low switching energy. In fact, all-optical demultiplexers (up to data speed at $250 \mathrm{~Gb} / \mathrm{s}$ ), ${ }^{1}$ all-optical logic gates $(40 \mathrm{~Gb} / \mathrm{s}){ }^{2}$ and wavelength converters $(65 \mathrm{~nm}$ wavelength shift ${ }^{3}$ have been demonstrated using SLAs as the nonlinear elements. Also, all-optical monolithically integrated demultiplexers $(40 \mathrm{~Gb} / \mathrm{s})^{4}$ and add/drop multiplexers $(20 \mathrm{~Gb} / \mathrm{s})^{5}$ using SLAs have been developed. Since many SLA switching devices consist of fiber, ${ }^{6,7}$ it is important to understand whether the chirp from the fiber will induce serious pulse distortion in SLAs.

For high speed SLA switching devices $(>200 \mathrm{~Gb} / \mathrm{s})$, subpicosecond pulses are required. Since the spectral width becomes wider than $5 \mathrm{~nm}$ for pulses shorter than $500 \mathrm{fs}$, the semiconductor amplifier gain dispersion may introduce some pulse distortions. It was studied recently that for the linear propagation, gain dispersion and the associated linear refractive index caused femtosecond pulse breakup in SLAs. ${ }^{8}$ On the other hand, it was found that spectral effects from the gain dispersion strongly influenced the ultrafast gain dynamics measured in the pump-probe technique. ${ }^{9}$ The subpicosecond pulse propagation has been studied in the spectral domain. ${ }^{10}$ However, the effect from carrier dynamics and gain dispersion on subpicosecond pulses in the temporal domain has not been directly studied.

In this letter, we present both experimental and numerical results of subpicosecond pulse propagation in a fiber and SLA combined system measured in the time and frequency domain. We find that pulse propagation in SLA is very sensitive to initial chirp created in the fiber. Also, we observe pulse broadening by a factor of two, and temporal side lobes appear in the cross correlation. The pulses are in the soliton regime in the fiber, and the SLA is under strong gain satura-

${ }^{\text {a)} E l e c t r o n i c ~ m a i l: ~ y k a o @ e n g i n . u m i c h . e d u ~}$ tion conditions. Pulse propagating in a SLA without fiber is also conducted for comparison. To identify the mechanisms which give rise to the pulse distortions, we numerically solve the propagation equations including gain change from carrier depletion, gain change from carrier heating, two photon absorption (TPA), three self-phase modulation (SPM) terms associated to the above gain changes, and gain dispersion. From the simulations, we find that the pulse broadening is due to TPA and the pulse breakup is caused by the interaction between the gain dispersion and frequency chirp. The strong pulse broadening and breakup may degrade performance of SLA devices for subpicosecond applications requiring cascadibility.

The experimental setup is shown in Fig. 1. We use a passively mode-locked KCL:Tl color center laser to generate 650 -fs pulses at a center wavelength of $1542 \mathrm{~nm}$ (nearly transform limited Gaussian pulses with $\Delta t \Delta \nu \approx 0.45$ ). A small portion of the laser output is split by a half-wave plate and a polarization beam splitter to serve as a reference beam for the cross correlator. The power in the fiber is about 0.9 of the soliton power, ${ }^{11}$ and the soliton length ${ }^{11}$ for 650 -fs pulses is $6.5 \mathrm{~m}$. We set the incident pulse polarization to TE using a fiber polarization controller. The light is coupled into the SLA by a short microlensed fiber with the coupling efficiency about $15 \%$. To filter out any light guided by the cladding, we use a spatial filter, which is placed $2 \mathrm{~m}$ away from the SLA. The output pulses are examined by a cross correlator and a spectrometer.

The SLA used is a $500 \mu \mathrm{m}$ long, channeled MESA bur-

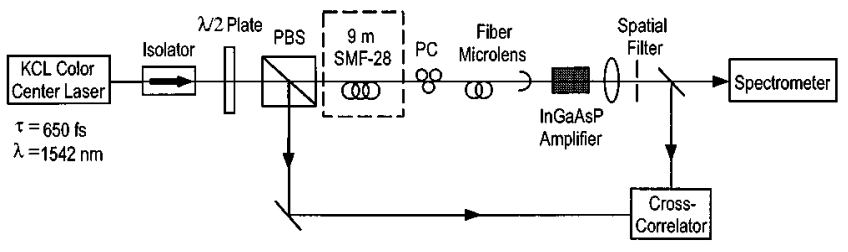

FIG. 1. Experimental setup for the study of pulse propagation in SLA. (PBS $=$ polarization beam splitter, $\mathrm{PC}=$ polarization controller, and $\mathrm{PMT}$ $=$ photomultiplier tube). 

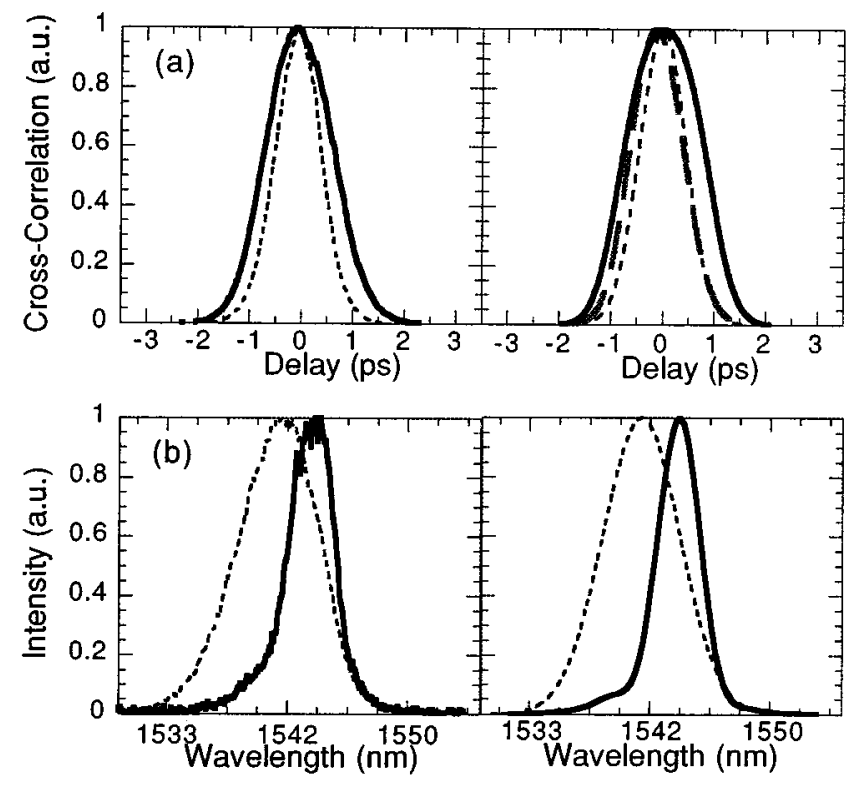

FIG. 2. The (a) cross-correlations and (b) optical spectra of the pulses before (dotted line) and after (solid line) propagation in SLA. The corresponding simulation results are shown on the right. The dashed curve is the output pulse cross-correlation when the TPA term in the simulation is turned off.

ied heterostructure $(\mathrm{CMBH})$ amplifier grown by metalorganic vapor phase epitaxy (MOVPE) with an antireflection coating on one facet. The active region consists of five 107$\AA$-thick $1.6 \mu \mathrm{m}$ InGaAsP quantum wells layered between 64- $\AA$-thick $1.3 \mu \mathrm{m}$ InGaAsP barriers. All the pulse propagation experiments are performed at the injection current of 70 $\mathrm{mA}$, which corresponds to a small signal gain of $\sim 13 \mathrm{~dB}$. The saturation energy [defined as $W_{s}$ in Eq. (2)] of the gain in SLA is about $10 \mathrm{pJ}$. Our laser center wavelength is at the shorter wavelength side of the gain spectrum with respect to the gain peak at $70 \mathrm{~mA}$.

We first conduct a linear pulse propagation to examine the effect of group-velocity dispersion (GVD) on 650-fs pulses. The input pulse energy coupled into the SLA is about $100 \mathrm{fJ}$. The cross correlation of the output pulse shows negligible change; therefore, we conclude that the GVD can be neglected for the 650 -fs pulses.

Figure 2 shows the experimental results for the 650-fs pulses propagating only through the SLA without the $9 \mathrm{~m}$ of fiber. The input pulse energy is about $12 \mathrm{pJ}$, which strongly saturates the SLA gain. Compared to the linear propagation case, we observe pulse broadening, and the broadening increases in proportion to the input power. Because of its power dependence, the broadening is most likely from TPA. We decorrelate the cross correlation and obtain pulse broadening by a factor of two. The red shift in the optical spectrum is mainly caused by the strong gain saturation from carrier depletion and the gain dispersion.

Figure 3(a) shows the cross correlation and optical spectrum of the output pulse after propagating through a $9 \mathrm{~m}$ of standard fiber followed by the SLA. The Gaussian pulses from color center laser evolve into hyperbolic secant pulses after propagating through a $9 \mathrm{~m}$ of fiber. The pulse width becomes $770 \mathrm{fs}$, and the time bandwidth product is about 0.38 (it is 0.315 for transform limited sech pulses). This
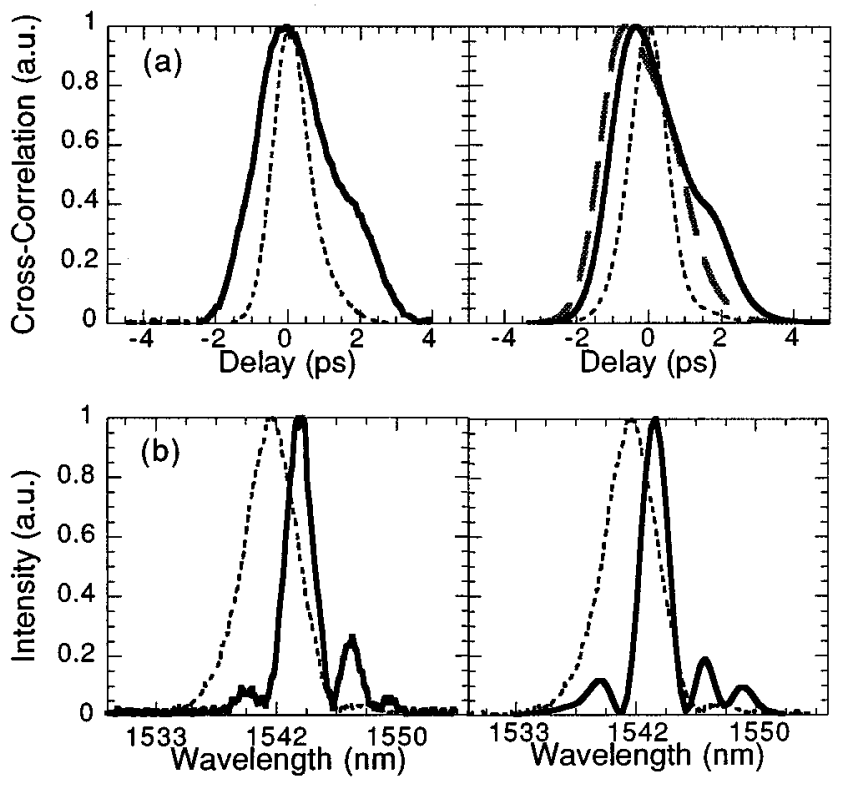

FIG. 3. The (a) cross-correlations and (b) optical spectra of the pulses before (dotted line) and after (solid line) propagation in a fiber and SLA combined system. The corresponding simulation results are shown on the right. The dashed curve is the output pulse cross-correlation when the gain dispersion terms in the simulation are turned off.

chirped pulse then propagates through the SLA. A second peak appears in the cross correlation for this case, and the peak becomes more observable when the input power increases. The power dependence indicates that the second peak is caused by nonlinear carrier dynamics in SLA. However, because pure frequency chirp cannot affect pulse shape in the time domain, the extra distortion should be caused by the gain dispersion, which couples the frequency chirp to a time domain modulation. Reference 10 shows that the addition of three types of SPM is complicated because of their different recovery times. The presence of initial chirp from the fiber makes the resultant SPM more complicated and introduces pulse breakup through the gain dispersion. In fact, this complicated addition also results in several peaks observed in the optical spectrum (Fig. 3).

To confirm our explanations for the experimental results, we numerically solve a propagation equation, which is similar to the equation described in Ref. 10:

$$
\begin{aligned}
\frac{\partial}{\partial z} V(t, z)= & \left(-\frac{\beta}{2 A}+i \frac{\omega_{0}}{C A} n_{2}\right)|V(t, z)|^{2} V(t, z) \\
& +\left(\frac{1}{2} g_{N}\left(t, \omega_{0}\right)\left(1+i \alpha_{N}\right)\right) V(t, z) \\
& +\frac{1}{2} \Delta g_{C H}\left(1+i \alpha_{C H}\right) V(t, z) \\
& -\left.\frac{i}{2} \frac{\partial g}{\partial \omega}\right|_{\omega_{0}} \frac{\partial}{\partial t} V(t, z)-\left.\frac{1}{4} \frac{\partial^{2} g}{\partial \omega^{2}}\right|_{\omega_{0}} \frac{\partial^{2}}{\partial t^{2}} V(t, z) .
\end{aligned}
$$

The terms on the right hand side of Eq. (1) are TPA, the corresponding nonlinear refractive index $n_{2}$, the gain saturation from carrier depletion, the associated index change 
TABLE I. Simulation parameters.

\begin{tabular}{|c|c|c|}
\hline $\begin{array}{c}\text { Pulse } \\
\text { parameters }\end{array}$ & $\begin{array}{c}\text { SLA } \\
\text { parameters }\end{array}$ & \\
\hline$\tau=650 \mathrm{fs}$ & $A_{\mathrm{eff}}=3 \mu \mathrm{m}^{2}$ & $L=500 \mu \mathrm{m}$ \\
\hline$\lambda_{0}=1542 \mathrm{~nm}$ & $n_{2}=-6 \times 10^{-13} \mathrm{~cm}^{2} \mathrm{~W}^{-1}$ & $\beta=80 \mathrm{~cm} \mathrm{GW}^{-1}$ \\
\hline$E_{\text {in }}=12 \mathrm{pJ}$ & $\begin{array}{l}W_{s}=10 \mathrm{pJ} \\
h_{1}=0.3 \mathrm{~cm}^{-1} \mathrm{pJ}^{-1} \\
\partial g / \partial \omega=0.95 \mathrm{fs} \mu \mathrm{m}^{-1}\end{array}$ & $\begin{array}{l}\tau_{C H}=600 \mathrm{fs} \\
h_{2}=300 \mathrm{~s} \mathrm{~cm}^{-1} \mathrm{pJ}^{-2} \\
\partial^{2} g / \partial \omega^{2}=-134.9 \mathrm{fs}^{2} \mu \mathrm{m}^{-1}\end{array}$ \\
\hline
\end{tabular}

through the linewidth enhancement factor $\alpha_{N}$, an addition gain reduction from carrier heating effect, the associated index change through $\alpha_{C H}$, the linear gain dispersion and the second order gain dispersion. We do not include GVD and spectral hole burning in our propagation equation because they are not significant for the $650-\mathrm{fs}$ pulses. The gain changes from the carrier depletion $g_{N}(t)$ and the carrier heating $g_{C H}(t)$ are expressed as

$$
\begin{gathered}
g_{N}(t)=g_{0} \exp \left(-\frac{1}{W_{s}} \int_{-\infty}^{t}|V(s)| 2 d s\right), \\
\Delta g_{C H}(t)=-\int_{-\infty}^{t} e^{-s / \tau_{C H}\left[h_{1}|V(t-s)|^{2}\right.} \\
\left.+h_{2}|V(t-s)|^{4}\right] d s,
\end{gathered}
$$

where $g_{0}$ is the small signal gain, $W_{s}$ is the saturation energy, $h_{1}$ is the contribution from stimulated emission and free carrier absorption, and $h_{2}$ is the contribution from TPA.

In order to investigate the case of pulse propagation in both fiber and SLA, we first obtain the initial chirp information by numerically solving the nonlinear Schrödinger equation $^{11}$ for pulse propagation in a $9 \mathrm{~m}$ of fiber. The first two orders $\left(c_{1}\right.$ and $\left.c_{2}\right)$ of the chirp in its Taylor series are described as:

$$
\begin{aligned}
V(t) & =\operatorname{sech}(t) \times e^{-i / 2\left(c_{1} t^{2}+c_{2} t^{3}\right)} ; c_{1}=-0.23, c_{2} \\
& =-0.011 .
\end{aligned}
$$

Here we use normalized unit $t=t / t_{0}^{2}$, and $t_{0}$ is the full width half-maximum of the pulse divided by 1.763 . Because propagation in fibers is well understood, we are able to reproduce the pulse shape and have confidence in the chirp extracted from the simulation.

The simulation results of pulse propagation are illustrated on the right-hand side of Figs. 2 and 3. The parameters used in the simulation are shown in Table I, where the input pulse parameters are the same as our experimental conditions and the SLA parameters are chosen to obtain the best fit for both experimental results. We are not only able to reproduce the pulse shape and optical spectra for both cases, but we also obtain the pulse broadening factor within $10 \%$. The good agreement between the experimental and simulation results confirms the validity of Eq. (1) and the parameter values in Table I. To verify that pulse broadening is primarily from TPA, we turn off the TPA parameter in Eq. (1) and show the result by the dashed line in Fig. 2. Only an asymmetric shift in the time domain from the gain saturation is observed without TPA. Also, to illustrate that gain dispersion is responsible for the extra peak in the cross correlation of Fig. 3, we turn off the gain dispersion term in Eq. (1) and the resultant dashed curve shows only pulse broadening. This proves that gain dispersion can actually transfer the frequency chirp to time domain modulation and GVD effect in SLA is not playing a role in pulse shaping for 650-fs pulses. By turning off either one of the gain dispersion terms in the simulation, we find that the distortions are mainly caused by the linear gain dispersion.

In conclusion, linear gain dispersion in SLA can convert frequency chirps to temporal distortions for subpicosecond pulses. We observe pulse broadening by a factor of two due to TPA for pulse propagating in SLA under strong gain saturation condition. We also verify through simulation that the pulse breakup observed in a fiber and SLA combined system is caused by the interplay between the gain dispersion and frequency chirp. Both the pulse broadening and breakup will degrade the cascadability of SLA logic gates, or increase the bit error rate of SLA demultiplexers.

This work at the University of Michigan is sponsored by the National Science Foundation and DOD. The authors would like to thank Professor E. P. Ippen for helpful discussions.

${ }^{1}$ I. Glesk, J. P. Sokoloff, and P. R. Prucnal, Electron. Lett. 30, 339 (1994).

${ }^{2}$ N. S. Patel, K. A. Rauschenbach, and K. L. Hall, OSA Tech. Dig. Ser. 9, 8 (1996).

${ }^{3}$ J. Zhou, N. Park, J. Vahala, M. A. Newkirk, and B. I. Miller, IEEE Photonics Technol. Lett. 6, 984 (1994).

${ }^{4}$ E. Jahn, N. Agrawal, M. Arbert, H.-J. Ehrke, D. Franke, R. Ludwig, W. Pieper, H. G. Weber, and C. M. Weinert, Electron. Lett. 31, 1857 (1995).

${ }^{5}$ E. Jahn, N. Agrawal, H.-J. Ehrke, R. Ludwig, W. Pieper, and H. G. Weber, Electron. Lett. 32, 216 (1996).

${ }^{6}$ J. P. Sokoloff, P. R. Prucnal, I. Glesk, and M. Kane, IEEE Photonics Technol. Lett. 5, 787 (1993).

${ }^{7}$ M. Eiselt, W. Pieper, and H. G. Weber, IEEE Photonics Technol. Lett. 13, 2099 (1995).

${ }^{8}$ S. Hughes, A. Knorr, and S. W. Koch, Opt. Lett. 21, 1052 (1996).

${ }^{9}$ J. Mork and A. Mecozzi, Appl. Phys. Lett. 65, 1736 (1995); J. Mork, A. Mecozzi, and C. Hultgren, Appl. Phys. Lett. 68, 449 (1996).

${ }^{10}$ M. Y. Hong, Y. H. Chang, A. Dienes, J. P. Heritage, and P. J. Delfyett, IEEE J. Quantum Electron. QE-30, 1122 (1994).

${ }^{11}$ G. P. Agrawal, Nonlinear Fiber Optics (Academic, San Diego, 1995). 